\title{
SEEDS OF HAPPINESS, OR THE MIND'S NETWORKS AND HAPPINESS Interview by Uliana
}

\author{
Lushch-Purii
}

In Happiness And Contemporary Society : Conference Proceedings Volume (Lviv, March, 20-21, 2021). Lviv: SPOLOM, 2021. P. 17-24. https://doi.org/10.31108/7.2021.1

ISBN 978-966-919-697-2 


\section{ARDEN John \\ PhD, Kaiser Permanente Medical Center; University of New Mexico \\ (Santa Fe, New Mexico, USA)}

\section{SEEDS OF HAPPINESS, OR THE MIND'S NETWORKS AND HAPPINESS Interview by Uliana Lushch-Purii}

My I am very happy to be a part of the conference "Happiness and Contemporary Society", it's a delight and an honor for me; I believe this is especially relevant during global pandemic. I am going to talk about how to survive and how to keep from getting unhappy during not only the restrictions period but also the post-COVID period, because many people will be experiencing lingering symptoms of COVID that we refer to as long COVID. So the experience of life satisfaction is incredible relevant with regard to how we take care of ourselves, how we maintain health and how we appreciate our lives. We also know that your age has a lot to do with your capacity to engage in some kind of level of satisfaction: older people, middle-aged people are a little bit more able (as you know from your research on the impact of quarantine restrictions on the Ukrainians' happiness) to maintain a sense of life satisfaction and happiness versus those young people that miss out on many of the networking experiences, social experiences that we all love during our teenage years, our 20 s and early 30 s. So I am going to try to weave in some of those comments throughout the presentation.

There are the factors that seem to be important for sustainable happiness from the foundational perspective, in other words you need to practice these factors on a daily basis no matter what's going on with you: you're locked in to your household, you can't get out during quarantine but you still need these five healthy factors that I've encoded in the mnemonic SEEDS.

You need to plant and cultivate these "SEEDS" on a daily basis. Some people say, "I've got a couple of them in place and that's should be ok". No, you need all five healthy factors that you engage in on a daily basis. They are: 1) social connectedness; 2) exercise (aerobic exercise, meaning to get the heart bumping); 3) education (learning, remaining curious about everything in the world); 4) diet (which is critical because you can undermine your immune system's stability if you maintain a poor diet); 5) sleep (a poor sleep architecture is also undermining the immune system's stability). They all have psychoneuroimmunological, epigenetic and neuroscience research backing. Psychoneuroimmunology that studies the interaction between the mind, the brain and the immune system. Epigenetics studies how to turn on the genes you want turned on and how to turn off the genes you don't want on.

The first one - social connectedness - is rather paradoxical, because what we are required to do to keep us all safe is social distancing. I good friend of mine has recommended, and I totally agree with him, that we should call it physical distancing, not social distancing. In other words, what we are doing right now, during Zoom conference, is social connectedness: I'm seeing your face, you're seeing my face, you're nodding as I'm talking, there's a connectivity here that is so critical. So during the period of pandemic and the post-pandemic we still need to maintain and to cultivate these social connective experiences and other four healthy factors. 
Just to put this in perspective, we know that some of our grandparents anticipated that we would be talking about how doing things in our lifestyle affects our bodies and our brains. Of course, a hundred years ago people had no idea about how this all worked, but they just hypothesized. Sigmund Freud: "We must recollect that all of our provisional ideas in psychology will presumably one day be based on an organic substructure". William James: "The act of will activates neural circuits".

More recently, a Noble laureate Eric Kandel has suggested that actually lifestyle has a huge effect on genes expression and the way your brain operates: "Psychotherapy works by producing changes in gene expression that alter the strength of synaptic connections". In other words, what you think about how you feel, what you do changes your gene expression. It's very critical to the stability of your capacity to generate happiness.

With regard to epigenetics, we had about 20 years ago an international effort called "The Human Genome Project" and we thought that we were going to have probably hundred thousand genes, it turned out we discovered roughly 24000 . There are other species that have only 24000 genes, one of them is a warm. So warm has the same number of genes. Maybe, we should consider what a gene is.

Gene is a section of your DNA that codes for amino acids that later make protein, that's all what genes do. You do not have a gene for happiness. You don't have a gene for depression or anxiety. If that was the case, think about warms going to psychiatric clinics for psychotherapy because they have panic disorders or schizophrenia. That doesn't happen. Genes code for amino acids that later turn into protein. How much of our DNA does that? 2\%. That means, $98 \%$ of our DNA are not genes. We used to call it "junk DNA" (non-coding) and the $2 \%$ thought to be the important one. But it occurred, our $98 \%$ help gene expression which means that we are adaptable by our lifestyle. A warm is almost all genes in the DNA. We have grater adaptability than warm. We basically change our DNA or rather our gene expression by our lifestyle.

Our telomeres which also are comprised by non-coding DNA ("junk DNA") change because of our lifestyle. Telomere caps the ends of your chromosomes. If you want to get ill, to get COVID-19 and do not want to recover quickly, shorten your telomeres. If you want to be healthy, resistant, resilient and to recover quickly, keep your telomeres strong - lifestyle is the way to do it. That's why we pay attention to the things that undermine our capacity to keep healthy. There are factors that make you more vulnerable by shortening telomeres: aging, cardiovascular disease, obesity, smoking, diabetes, social isolation, poor diet, no exercise, poor sleep. The first in the list - aging we just can't stop doing, you can slow it down or you can speed it up. But all the other ones are very strongly affected by lifestyle and some of them are lifestyle factors. So what we need is the five healthy factors, encoded into the SEEDS mnemonic, that give you the foundation to be happy, maintain a high level of life satisfaction and so on.

A few words on the immune system because we are so compelled to understand how the immune system is operating, relative to COVID-19 in particular. There is a lot of immune cells in your gut, but you also have the immune system in your brain. So if you have chronic inflammation which is really a part of the sequelae of symptoms or causal factors for long COVID than what you have is depression and the feeling of unhappiness, you're not able to generate happiness. That's why people with COVID and long COVID have what they refer to as brain fog (clouding of consciousness).

What is going on with this immune system in a brain? If you have immune overreaction anywhere in your body, you are going to turn on these immune cells in your 
brain, specifically we are talking about the glial cells that are called micro-glia (there are four different types of glial cells). Micro-glia or immune cells in the brain can respond to pro-inflammatory cytokines by spidding out more of them. So if you have long COVID and you do not climb out of the long COVID by practicing the five healthy factors, you could accelerate the propensity to develop dementia much more quickly than other people. That's the shocking part of what we are experiencing now as the world population!

So what we need to do is to turn down chronic inflammation. COVID-19 itself is related to what we call the cytokine storm: that's pro-inflammatory cytokines just blasting all over the place and what we need to do is to get the inflammation down in the overactive immune system. Chronic COVID, long COVID is chronic inflammation and we need to get it down. When you have chronic inflammation, your capacity to generate happiness diminishes: you get lethargic, you can't generate positive thoughts, you get brain fog and you just don't see any positive things going on around you. So what we need to do is to get handle on the chronic inflammation. We do know is that all research related to depression, prior to COVID, was centered around chronic inflammation. Isn't it interesting that after all these studies dating back to the late 1990s up until roughly about a year ago we were trying to target chronic inflammation to help with depression? In fact, many of these drugs (as Prozac and others) were actually seem to not just affect serotonin but also act as anti-inflammatory agents. By the way - and I feel compelled to say this - are trying to fight COVID with antibiotics. It's not a bacterium. Unless you have a bacterial infection. With antibiotics you wipe out bacteria of your gut and you'll have inflammation.

We want to get our thinking process down. There is no one thing called thought. There are many different mental operating networks that we have to pay attention to. There is no one thing called mind, it's really states of mind generated by specific different brain areas that are jumping around. I might fade off in my default mode network because I'm not as centered and focused on the here and now, I might feel a little differently emotionally because my emotional network is sort of bogged down, perhaps, with chronic inflammation. So we know that these brain networks are critically involved in different types of thought and we need to keep them in balance. With COVID, we know that people have a tendency to be a little bit too much in default mode network, in other words they are not here and now, it's hard for them to focus on the harder things to do - be present and be goal-directed which has a lot to do with your capacity to generate happiness. Keeping these mental operating networks in balance is part of what we prefer to as mental health.

We also have a library of experiences that we code into our memory systems. They are super-complex, we can spend several hours just on the memory systems, but nevertheless I just want to highlight that these mental operating networks derive their information from the library of coded information within our brain and body.

A little bit about how the brain hemispheres work with happiness or depression. We know that the two hemispheres generate emotions differently. People who are to be more right hemisphere oriented tend to be more into withdrawal patterns and avoidance patterns and tend to be more anxious and depressed. We know that trauma victims tend to withdraw, avoid and get more of these icky feelings of not only depression but also anxieties. What we also know through a lot of the evidence based practice research related to psychotherapy, that getting people doing things, engage in life activates the left prefrontal cortex and activates positive experiences. In other words, avoidance and 
withdrawal generate negative emotions, whereas positive emotions are generated through activation and approach behaviors.

The left hemisphere is better able to activate positive emotions and suppress sadness. So what you can say to a client that you are working with, if you're a counselor therapist, is that when you are overwhelmed with anxiety and depression it is best to shift from the big picture (that the right hemisphere creates) to the small and to do something that approaches a goal in a piecemeal, incremental manner (the left hemisphere is responsible for engagement in activity here and now). That is the way to climb yourself out of anxiety and depression: I am going to do this now, then I'll do that, in small pictures. It's very important to note that I am not talking about shutting down the right hemisphere, I'm talking about balancing it with the left hemisphere.

Left hemisphere is better able to generate feelings of life satisfaction; and if you are curious about anything you explore it through activation of your left prefrontal cortex. Let's speak about the positive part of the right hemisphere: let's take humor, for example. If you get your right hemisphere knocked out, you lose your sense of humor. So what is humor? Humor is being able to stand back and laugh at something. The best type of humor that you could possibly have - and these are people who are the happiest are the people who can laugh at themselves in a liberating way. I don't mean make fun of yourself: "Oh, I'm so terrible". No, I'm talking about find things funny, find yourself funny. If you laugh at somebody else it's not happiness, that's not humor, that's meanness. The right hemisphere gives you a capacity of humor if you balance it with the left one as well.

SEEEDS of happiness. The first one is social connectedness, engagement (S-eeds). Why is it so important? Because we are a social creature. All of us on this planet roughly before 11000 years ago we were hunters-gathers. We moved around 10 miles a day. If you get kicked out of a group, you lose any sense of connectivity, you get eaten by a lion but you also get super depressed. There is psychoneuroimmunological explanation, there is an activation of your immune system - that is really critical - if you are socially connected. If you have isolation, if you are disconnected from people, if you feel that there is nobody that cares about you, your immune system goes flat. So forget the social distancing, it's physical distancing because we want to keep our sense of connectivity. The best type of social connectivity we possibly can have is the capacity to have empathy and compassion for one another. That is the antidepressant. A lot of people get a sense of happiness by giving to other people: you actually generate happiness within yourself because there are areas of the brain that are very strongly involved with the capacity for empathy like the anterior cingulate and others. Being able to give to others actually is a giving to yourself, so giving is really receiving. Those people who are selfish and can't give to other people, actually experience unhappiness and deplete their immune system.

We also shrink areas of the brain, like the temporal-parietal junction (TPJ) which is on the right side in the intersection of parietal and temporal lobe. You shrink that area with loneliness. Those of us in the helping health care, especially mental health professionals, always exercise in this area of the brain. We also know that your capacity to generate the dopaminergic circuits - dopamine from the ventral tegmental area to anticipate any kind of positive experience - decreases with loneliness. So social connectedness is very important for the feelings of life satisfaction.

Let's move to exercise (s-E-eds) which is so incredible important to generate networks in the brain. People used to move 10 miles a day as I've mentioned above. 
Who moves 10 miles a day right now? Well, not many people do that unless you're in a car. We are actually devolving right now; we are not doing what our body evolved to do. We are now sitting around computers all the time. Moving on a regular basis is actually anti-inflammatory. One of the major factors related to COVID-19 is chronic inflammation. You can activate anti-inflammatory cytokines - myokines - through movement of your muscles as well as your cardio-vascular system.

One way to measure inflammation is through protein that is released through various organs including your liver. C-Reactive Protein is a measure of inflammation. The more vigorous your exercise is or cardio-vascular boosting of your exercise, the lower inflammatory networks are, even if you don't feel like it. So we need to get people moving. It's also related to your capacity to generate positive emotions and happiness. So exercise is the most powerful antidepressant that we have and you don't stick it in a pill. The multiple studies throughout the world have demonstrated that exercise is the fundamental thing we want to get going with people who are depressed.

Other networks that are critically important. There is a magical organic fruitalizer - Brain Derived Neurotropic Factor (BDNF) that is very involved in neurogenesis and neuroplasticity that gets released with exercise. Also your capacity to use energy in the form of glucose through Insulin-like Growth Factor (IGF-1) as well as other neurotropic factors that help your brain get rewired, as well as keeping the capillaries (little blood vessels) in your brain very flexible so you don't have aneurisms. Vascular Endothelial Growth Factor (VEGF) that impacts neurogenesis is also improved with exercise. So due to exercise you have a happy brain, brain capable of happiness.

Next is education (se-E-ds). It doesn't necessarily mean to have fancy degrees; you can just constantly learn. When you are learning, you are connecting neurons that weren't connected before, that's called synaptic plasticity. You have 100 billion neurons in the brain and 10000 connections with other neurons; the more connectivity you have, the more capable you are of different appreciations for life around you. Meaning, the more subtleties you can experience in a positive way, the happier you are. If you only derive your sense of happiness from one thing, you're stuck because you can't always get that one thing. Moreover, you can't regenerate dopamine receptor sites if you're only focused on one thing - it is called addiction.

Happiness and the resistance to any kind of addiction necessitates that we derive pleasure from subtlete interactions. Moderate level of life appreciations is much better; there is a term in Buddhism - the Middle Path. That means you need to connect these different neurons to have much more complex feeling of life appreciation.

We are looking at how the brain in new dendritic spines as you learn. So you have to get out of your comfort zone to be able to do that. We know for 100 years now due to the study by Yerkes Dodson that you have to be uneasy to be able to remember stuff. Just learning stuff that you like doesn't build brain that is capable of all these experiences. What you need to do is to step out of your comfort zone to build a much more complex brain. You need to be a little uneasy.

The next healthy factor is diet (see-D-s), it's essentially brain food. Lockdown conditions decrease our problem solving capacity. You need to feed your brain network with fuel to develop the capacity of these kinds nimble cognitive experiences and to be able to generate moods that are positive. Unfortunately, we have been engaged in undermining our capacity for these brain networks in the standard Western diet. We're now seeing an actual shrinkage of the brain in particular areas because of the Western diet (simple carbohydrates, fried food etc.). You've got all sorts of problems, not only 
the lifestyle diseases, metabolic syndrome, type II diabetes, but also the shrinkage of the left hippocampus, more negative low density cholesterol, increased blood pressure etc.

The gut bacteria aspect is really very important to diet. If your diet is imbalanced, you can get inflamed gut and not being able to get out of long COVID or get more vulnerable to COVID-19. If you feed the firmicutes (a category of gut bacteria) by simple carbohydrates and other things and don't maintain the Mediterranean diet you're going to have inflamed gut, have more fat cells, more propensity to develop diabetes type II, cardiovascular disease etc. or minimize the amount of bacteriodetes that is part of what lean people generate because they are eating more fruits and vegetables. What we want to do is lean towards the Mediterranean diet, minimize the standard Western diet processed foods.

By the way, antibiotics wipe out all gut bacteria. If you need to take it because you've got infection, not a virus, you need to build up your gut bacteria again with vital nutrients. Otherwise you are going to have a "leaky gut" because you have to much of pro-inflammatory cytokines activity going on and that turns on the inflammation in your brain.

What we don't want to do is to keep this negative circle going because you get brain fog. Diet is a big part of this picture. Minimize simple carbohydrates, saturated fats, trans fatty acids, bad oils and exercise instead of sit around thinking you're too tired for it, because this cornucopia of brain nutrients is critical for your brain, including all the neurotransmitters that are made in your gut, the amino acids that you take in through your diet.

So let us move on to the last factor - sleep (seed-S) that is critically important for your immune system. During your sleep hours you are generating more immune system activity, assuming that you are maintaining a good circadian rhythm. To keep a healthy circadian rhythm, we need to go to sleep at the same time almost every night and get up at the same time. We need to maintain good sleep architecture. A good slow wave sleep - that's when your immune system rebuilds; if you don't get it, you end up groggy later in the day. Slow wave sleep is very different from Rapid Eye Movement sleep (REM sleep), which relates to a brain activity that is very much like what we generate right now as we go through our default mode network. Unfortunately, in the Western world, we are blocking a lot of our slow wave sleep by additives. People are taking over the counter drugs or prescribed drugs to get caught sleep but it's really a not good quality sleep. Benzodiazepines and Benadryl-like substances are the garbage of our health care system. You want dementia quick - block your slow wave sleep. You want not to get out of the COVID syndrome - block your slow wave sleep. Because there is the brain clearing process as well as activation of your immune system. Otherwise you're going to feel more fatigue, have brain fog and depression, incapable of generating happiness.

What happens during slow wave sleep is like a washing machine clearing your brain out of all the builds up of proteins like $\beta$-amyloid and others. We know that people who take additives for sleep are actually more prone to get Alzheimer's disease later on in life and more vulnerable to depression because they are incapable of generating happiness.

Try eating a light snack with complex carbohydrates before bed. Food rich with LTryptophan are advisable. Don't eat anything with sugar or salt. Avoid protein snacks before bed because protein blocks the synthesis of serotonin and as a result promotes alertness. 
Body temperature is also critical for a good sleep: you need to keep a cool body temperature throughout the evening. You can get to sleep with a nice duvet, a quilter or whatever but you are not going to be able to generate the deep sleep. So crack the window open, keep your blanket lighter - that is very important to keep a good sleep architecture. Otherwise you are going to wake up not feeling as peppy, positive and generating happiness.

To summarize, the five healthy factors need to be in place every single day - that is the foundation for happiness and physical health; and physical health has a huge amount to do with mental health.

Uliana Lushch-Purii, PhD, Associate Professor, Co-founder of The Ukrainian Institute for Happiness Research: Based on your personal observation, based on your psychiatric and psychological practice, how often do people come with a problem "I'm not feeling happy"? Or, maybe, happiness is not an issue at all?

The term they would often use is "unhappiness". So instead of saying "I'm not happy", people often say "I'm unhappy" or "life is not what it used to be", "nothing seems to really make me feel good anymore". There are different ways of saying the same thing; they don't necessarily say "I'm coming in to talk with you about happiness", but what they are talking about is all the factors that relate to happiness on the other side. I've been in practice for over 40 years, I've seen a lot of people. I think it's really important for us to bring things down to earth for our clients and to help our clients to understand rather that make it all esoteric. Do I get in to talk about myokines and proinflammatory cytokines and use all that terminology with my clients when they come and say "I'm unhappy"? No, I try to keep it really-really simple because if they are depressed and unhappy they are not going to remember stuff like that. They are just going to remember things like SEEDS mnemonic. I just say that you need the chemistry, the brain networks to be able to generate a foundation for happiness; and if you are undermining the foundation of happiness, like building a house on sand, with any little shake your whole house is going to crumble down. Over the years I tried to appeal to where a person's cognitive ability is at, what they are capable of thinking at that time, that's one of the reason for the SEEDS mnemonic - to keep it simple.

A state of happiness is not a constant experience, we have fleeting periods of happiness; and sometimes people who are suffering from illness and not so good mental health have a thought that they need to feel happy all the time. But who does feel happy all the time? Your moods are always fluctuating; and we have periods of feeling better and feeling a little less good. So if they can understand that happiness is not a constant. You are constantly revolving around different mental operating networks even if you are super into mindfulness, meditation, you still have periods of fluctuating thoughts and feelings. So people need to understand there is not a constant feeling of happiness but rather a waviness to subtle experiences of life appreciation we can also call happiness. There is no surge, because then people get into addictive modes with dopamine circuits that they can't maintain. So happiness is the Middle Path of subtle appreciation that is a hallmark of the capacity to maintain happiness.

Uliana Lushch-Purii: There is one observation that concerns the study our team run last year, and that was the study of the impact of quarantine restrictions on people's happiness. We have found out that a group of people over 45 years old and around 55-60 have more sustainable happiness which doesn't depend on some external factors and is not easily decreased by external circumstances. So even though they had lower income and felt discomfort because of quarantine restrictions, they still reported being happy, 
remaining happy. As for a group of young people 18-22 years old, this group was the most vulnerable, their happiness was the most vulnerable to external circumstances. So we assume that the reason might be the difference in values between these groups, happiness is defined by different things by people of these two groups. What reasons could you add from psychiatric, psychological or neuropsychological perspective?

Your research makes a whole lot of sense and that's what I would anticipate that the people who are middle-aged and above are providing a fairly good foundation and are able to generate happiness because they are able to differentiate and appreciate subtleties in their environment but also delay gratification too. What is that all about? It's about the prefrontal cortex. So when you talk about people of 18 to 22 years old, we do know that the prefrontal cortex isn't totally formed until the mid-20s for women and even later for man. So the capacity to delay gratification is really a big part of the prefrontal cortex development. While you were talking, I was thinking of what I would experience during this quarantine if I was let's say 22. When I was 22 I was travelling around the world through Asia, Middle East and came back when I was 23. I couldn't do that stuff during COVID-19 pandemic and I couldn't go hang out with friends and at the age 18-22 a person can't delay gratification, their life has been relatively short, so they can't really think "well, things will get better over a period of time because things are not so immediate". Developmentally people who are in that age group are more heavily impacted by the shutdown. Here is some other aspect that I think is really unfortunate, is that because of the need of physical distancing people and young people especially are using social media for communication and stay in front of monitor for a long time. The light of the monitor, when it is used late at night, diminishes our capacity to create a good sleep architecture. You need to use computer, but do not do it late at night!

Uliana Lushch-Purii: Thank you John for being a part of the conference because indeed the mission of this project is to popularize knowledge of happiness and to show people how to apply this knowledge to their daily practice and live a long, healthy and happy life.

Thank you and thank you for what you're doing the world service by your research and having conferences like this and that's a great honor to be part of it.

\section{REFERENCES}

1. Arden, J. (2014). The Brain Bible. A Plan to Stay Vital, Productive and Happy for a Lifetime. New York: McGraw-Hill Education.

2. Arden J. (2019). Mind - Brain - Gene. Toward Psychotherapy Integration. New York; London: W.W. Norton \& Company.

3. www.drjohnarden.com - website of Dr Arden 